Phase III randomised trial

\title{
Does an integrated boost increase acute toxicity in prone hypofractionated breast irradiation? A randomized controlled trial
}

\author{
Leen Paelinck $^{\mathrm{a}}$, Akos Gulyban ${ }^{\mathrm{b}}$, Ferenc Lakosi ${ }^{\mathrm{b}}$, Tom Vercauteren ${ }^{\mathrm{a}}$, Werner De Gersem ${ }^{\mathrm{a}, \mathrm{c}}$, Bruno Speleers ${ }^{\mathrm{c}}$, \\ Christel Monten ${ }^{\mathrm{a}, \mathrm{c}}$, Thomas Mulliez ${ }^{\mathrm{a}}$, Patrick Berkovic ${ }^{\mathrm{b}}$, Annick van Greveling ${ }^{\mathrm{a}}$, Frederik Decoster ${ }^{\mathrm{a}}$, \\ Philippe Coucke ${ }^{\mathrm{b}}$, Wilfried De Neve ${ }^{\mathrm{a}, \mathrm{c}}$, Liv Veldeman ${ }^{\mathrm{a}, \mathrm{c}, *}$

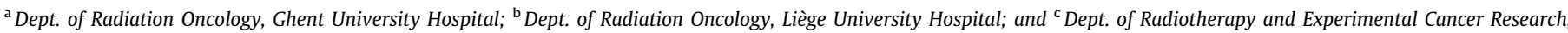 \\ Ghent University, Belgium
}

\section{A R T I C L E I N F O}

\section{Article history:}

Received 23 August 2016

Received in revised form 15 December 2016

Accepted 19 December 2016

Available online 3 January 2017

\section{Keywords}

Breast cancer

Whole-breast irradiation

Prone

Hypofractionation

Simultaneous integrated boost

\begin{abstract}
A B S T R A C T
Background and purpose: The safety of a simultaneous integrated boost (SIB) in combination with prone hypofractionated whole-breast irradiation (WBI) was investigated.

Materials and methods: 167 patients were randomized between WBI with a sequential boost (SeB) or SIB. All patients were treated in prone position to $40.05 \mathrm{~Gy}$ in 15 fractions to the whole breast. In the control arm, a SeB of $10 \mathrm{~Gy}$ in 4 fractions (negative surgical margins) or $14.88 \mathrm{~Gy}$ in 6 fractions (transsection) was prescribed. In the experimental arm a SIB of 46.8 or $49.95 \mathrm{~Gy}$ (negative and positive surgical margins, respectively) was prescribed.

Results: Patient age was the only significantly different parameter between treatment arms with patients in the SIB arm being slightly older. In both arms, 6/83 patients developed moist desquamation. Grade $2 / 3$ dermatitis was significantly more frequent in the SeB arm (38/83 vs $24 / 83$ patients, $p=0.037)$. In the SIB and SeB arm, respectively, 36 patients $(43 \%)$ and 51 patients $(61 \%)$ developed pruritus $(p=0.015)$. The incidence of oedema was lower in the SIB arm ( 59 vs 68 patients), but not statistically significant $(p=0.071)$

Conclusions: The primary endpoint, moist desquamation, was not significantly different between treatment arms.
\end{abstract}

๔ 2016 Elsevier Ireland Ltd. All rights reserved. Radiotherapy and Oncology 122 (2017) 30-36
Moderate hypofractionation in 15 or 16 fractions has been generally accepted as a valid alternative for the historical schedules of 25-30 fractions for whole-breast irradiation (WBI) after breast conserving surgery (BCS). With a median follow-up of 9.9 years, the UK START-B trial showed no significant difference in locoregional control between a hypofractionated schedule of $40.05 \mathrm{~Gy}$ in 15 fractions and a normofractionation scheme of $50 \mathrm{~Gy}$ in 25 fractions [1]. The Canadian schedule of $42.5 \mathrm{~Gy}$ in 16 fractions was also not inferior to the $50 \mathrm{~Gy} / 25$ fractions after 10 years of follow-up [2]. Aside from the obvious advantage of shortening the overall treatment time from 5 weeks to 3 weeks, both for the patient and the radiotherapy department, there might be some radiobiological advantages too. In the START-B trial, breast shrinkage, telangiectasia and breast oedema were significantly less frequent in the hypofractionation group. Better disease free survival and overall survival were also reported in the 15 fractions group,

\footnotetext{
* Corresponding author at: Ghent University Hospital, Dept. of Radiation Oncology, Radiotherapiepark, De Pintelaan 185, 9000 Ghent, Belgium.

E-mail address: liv.veldeman@uzgent.be (L. Veldeman).
}

due to less distant relapses. The superiority of hypofractionation on survival and cosmesis was not observed in the Canadian trial.

While moderate hypofractionation schemes are slowly replacing normofractionation schemes worldwide for WBI, the role of a boost dose after hypofractionation is still unknown. After normofractionation, a boost to the region at high-risk for relapse has been shown to improve local control [3]. In the START trials, about $40 \%$ of patients received a boost of $10 \mathrm{~Gy}$ in 5 fractions [1]. In the Canadian trial, boost treatment was not allowed, which might explain the higher rate of local relapse seen in the subgroup of patients with a high-grade tumour treated by hypofractionation [2]. While there is some discussion about the role of a boost in patients over 60 years, it seems not recommendable to omit the boost in younger patients [3]. A sequential boost ( $\mathrm{SeB}$ ) is typically delivered in 4 to 8 extra fractions which again prolongs the overall treatment time by 1-2 weeks. A so-called simultaneous integrated boost (SIB) technique allows delivering the boost simultaneously with the whole-breast treatment, avoiding unnecessary treatment prolongation. 
Increased dose conformality with a SIB has been reported in comparative SeB-SIB treatment planning studies [4-7]. The combination of a SIB with normofractionation schemes does not seem to increase toxicity either $[8,9]$. The trials on hypofractionated SIB treatment are scarce. Two non-comparative trials describe acceptable toxicity, cosmesis and quality of life $[10,11]$. One randomized controlled trial in 69 patients showed less toxicity in the SIB arm after 1 year of follow-up, while heart and lung function were not impaired [12]. Recently, the group of Sylvia Formenti published data on 400 patients treated to $40.05 \mathrm{~Gy}$ in 15 fractions to the breast, randomized between a daily concomitant boost of $0.5 \mathrm{~Gy}$ or a weekly $2 \mathrm{~Gy}$ boost. Acute grade 2 and long-term toxicity were comparable in both groups with a median follow-up of 45 months [13]. Long-term follow-up data of SIB treatment are limited, but local control after 5 year seems to be acceptable [14,15].

With the exception of the patients treated at the New York University School of Medicine [13,15], all patients in the abovementioned studies were treated in supine position. Although not generally accepted yet, prone position is an alternative for WBI with or without a boost. The merits of prone over supine position include: better avoidance of lung tissue (in all patients) and heart tissue (in the majority of patients) [16-18], better dose homogeneity with less hot spots and a favourable toxicity profile, especially in large-breasted females [19]. Disadvantages include the complex and uncomfortable setup and reduced reproducibility. Both centres involved in this trial, have experience with prone position in combination with hypofractionation for WBI without lymph node irradiation. The aim of this study was to investigate whether a SIB can safely be used in combination with prone hypofractionated WBI.

\section{Materials and methods}

\section{Patients}

As illustrated in the Consort diagram (Fig. 1), 168 patients were included after breast conserving surgery for early-stage breast carcinoma. 151 patients were treated at Ghent University Hospital (UZ Gent) and 17 at Liège University Hospital (CHU Liège). The study was approved by the Ethics Board of both hospitals and registered at clinicaltrials.gov (NCT01973634). Exclusion criteria were: mastectomy, lymph node irradiation, no boost dose planned according to the institution's practice guidelines, bilateral breast irradiation, patient unable to be treated in prone position, age $<18$ years, pregnant or breast feeding. At the beginning of the trial, patients with a left-sided tumour and European cup size A or B were also excluded because a higher heart dose was feared in prone position in this population of small-breasted patients [16,17]. From October 2013, all left-sided patients received prone breath hold [20] during treatment if mean heart dose was $\geqslant 2$ Gy or the maximum heart dose (D02) was $\geqslant 10 \mathrm{~Gy}$. D02 was defined as the dose received by $2 \%$ of the heart volume. An amendment to the protocol was approved by the Ethics Board, allowing patients with A and B cup to be included. For 1 patient allocated to the experimental treatment, radiation treatment was cancelled because axillary lymph nodes were detected on the planning CT. After axillary lymph node dissection, she did no longer meet the inclusion criteria of the trial because lymph node irradiation was required. This patient was treated at UZ Gent.

\section{Radiotherapy}

Patients were treated on a modified AIO Prone Breastboard at UZ Gent [19] and on the Sagittilt Prone Breast Solution at CHU Liège (both Orfit Industries, Wijnegem, Belgium). All patients were treated in prone position to $40.05 \mathrm{~Gy}$ in 15 fractions. The boost was delivered sequentially (84 patients) or simultaneously (83 patients) according to randomization. In the SeB arm the boost was delivered in 4 fractions of $2.5 \mathrm{~Gy}$ when negative surgical margins were achieved. In case of close or positive surgical margins, the boost dose consisted of $14.88 \mathrm{~Gy}$ in 6 fractions of $2.48 \mathrm{~Gy}$. Patients of the experimental arm received a SIB to the tumour bed of $3.12 \mathrm{~Gy}$ (negative surgical margins) or $3.33 \mathrm{~Gy}$ (positive surgical margins) per fraction to a total cumulative dose of $46.8 \mathrm{~Gy}$ or 49.95 Gy.

The clinical target volume for boost irradiation $\left(\mathrm{CTV}_{\text {boost }}\right)$ was defined by the radiation oncologist using all available preoperative and postoperative information like surgical clips, seroma, preoperative imaging, the surgical report and the histopathology report. For WBI the whole breast was delineated by the aid of a copper wire placed around the palpable breast tissue in prone position. A planning target volume for $\mathrm{WBI}\left(\mathrm{PTV}_{\mathrm{WBI}}\right)$ extending in air was created to account for swelling of the breast and respiratory movement. An inverse planned intensity modulated radiotherapy (IMRT) technique was used. During optimization a virtual bolus was used to assure skin flash [21]. For the final dose calculation (without the virtual bolus), dose was prescribed to the PTV $\mathrm{opt}_{\text {. This }}$ is an optimization structure created by removing the in-air part of the PTV ${ }_{\mathrm{WBI}}$ and the first $7 \mathrm{~mm}$ underneath the skin to remove the

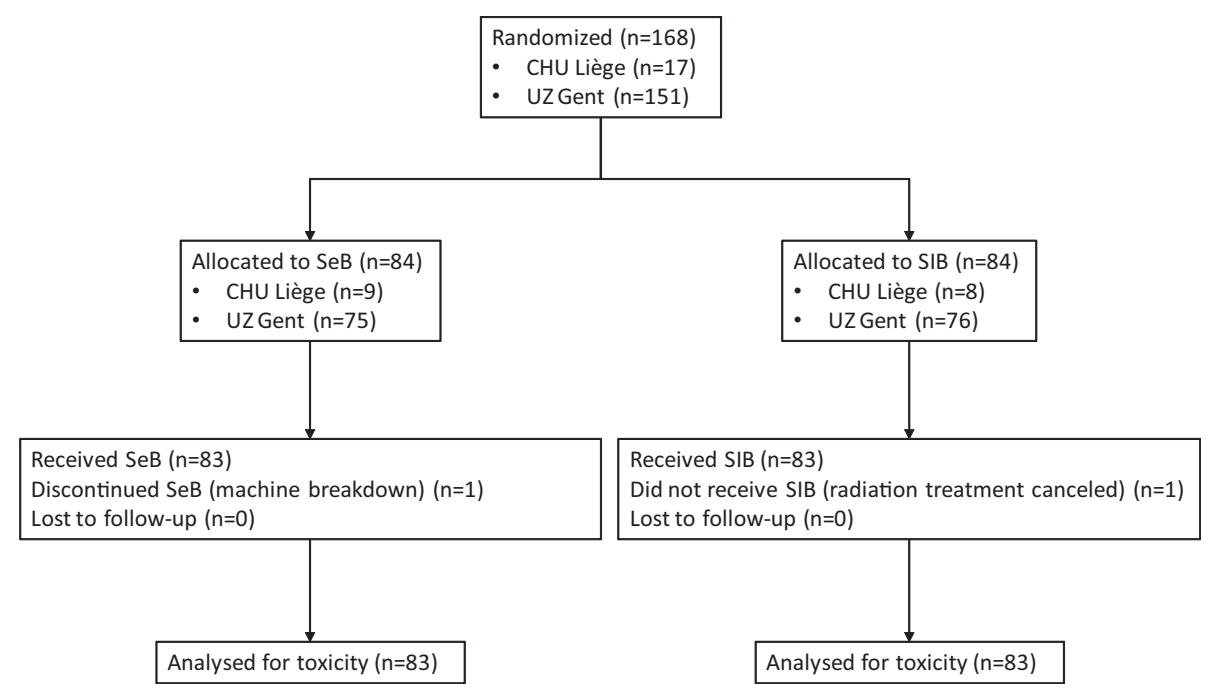

Fig. 1. CONSORT diagram. 
build-up region of photon beams [22]. In both treatment arms, the majority of patients received one medial and one lateral beam for WBI and 2-3 beams (mostly 1 beam with a table isocentre rotation) for boost irradiation. The 26 patients treated with breath hold were planned with a comparable intensity modulated arc therapy (IMAT) technique with very small $\left(2-3^{\circ}\right)$ medial and lateral arcs. IMAT was only used for practical reasons: faster delivery and therefore less breath holds needed than with IMRT.

In the SeB arm treatment was planned in 2 phases. First, a median dose of $40.05 \mathrm{~Gy}$ in 15 fractions was prescribed to the PTV while it was assured that $95 \%$ of the PTV opt $_{\text {received } 38 \mathrm{~Gy} \text { and }}$ no more than $5 \%$ received $42 \mathrm{~Gy}$. In the second phase, a median boost dose of $10 \mathrm{~Gy} / 4$ fractions or $14.88 \mathrm{~Gy} / 6$ fractions was prescribed to the PTV boost $_{\text {( }}\left(5 \mathrm{~mm}\right.$ isotropic expansion from $\mathrm{CTV}_{\text {boost }}$ ). Planning objectives included a D95 of $95 \%$ and a D05 of $105 \%$ of the prescription dose. D02 was defined as the dose received by $2 \%$ of a structure's volume. D95 was defined by the dose received by $95 \%$ of a structure's volume. For comparative analysis, dose parameters in the SeB arm were calculated on the summed dose distribution of the WBI phase and the boost phase.

For the patients treated with a breath hold technique a summed dose distribution was calculated on the breath hold CT [23]. A deformation field (DF) was generated between the breath hold CT and the shallow breathing CT using the Advanced Medical Image Registration Engine (ADMIRE) v1.10.01 (Elekta AB, Stockholm, Sweden) software package.

In the SIB arm, only one treatment plan was created for 15 fractions. A median dose of 46.8 or $49.95 \mathrm{~Gy}$ was prescribed to the $\mathrm{CTV}_{\text {boost }}$ with a dose gradient to $40.05 \mathrm{~Gy}$ in the first $2 \mathrm{~cm}$ around

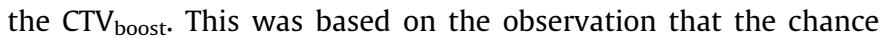
of additional tumour foci is highest in the first $2 \mathrm{~cm}$ around the reference tumour [24]. The part of the PTV opt more than $2 \mathrm{~cm}$ away from the $\mathrm{CTV}_{\text {boost }}$ was defined as a second optimization structure (optim structure, illustrated in Fig. 2) and here a median dose of 40.05 Gy was aimed for. Again, a D95 of 95\% and D05 of $105 \%$ of

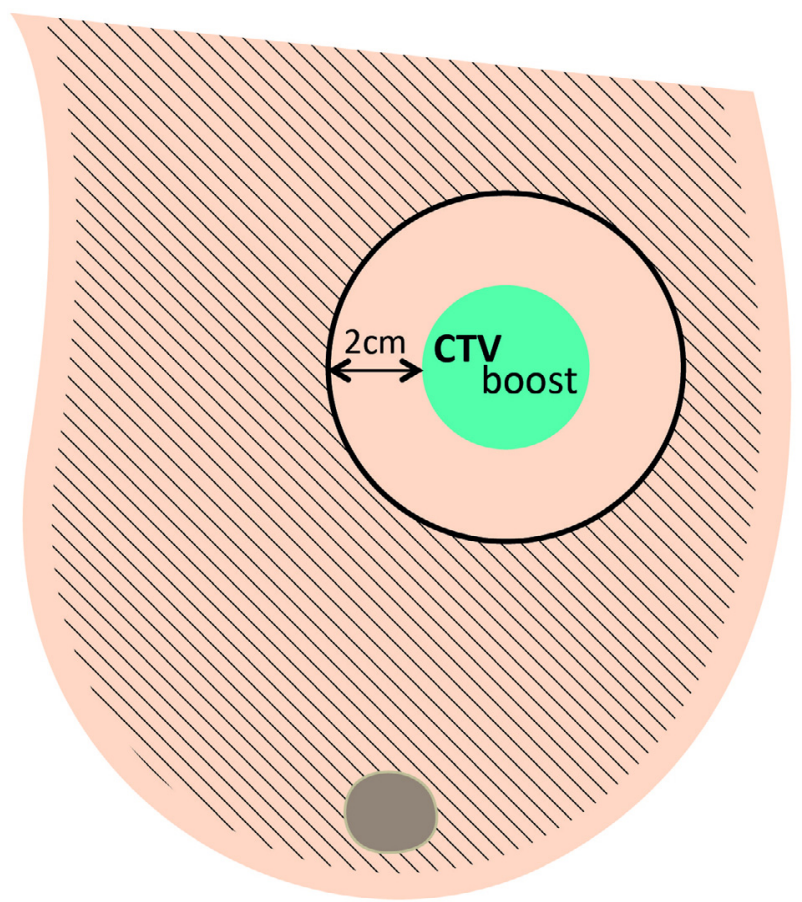

Fig. 2. Illustration of dose prescription in the SIB arm. Dose was prescribed to the CTV boost. A rim of $2 \mathrm{~cm}$ around the CTV boost was created for dose fall off. The remaining breast tissue, outside the rim, was defined as the optim structure (shaded area). the prescription dose were intended for the $\mathrm{CTV}_{\mathrm{boost}}$ and the optim structure.

Absolute doses in the plan were recalculated to equivalent doses in $2 \mathrm{~Gy}$ fractions (EQD2) for comparison. An $\alpha / \beta$ value of $10 \mathrm{~Gy}$ was used for acute toxicity and of $3 \mathrm{~Gy}$ for late side effects.

\section{Endpoints}

The primary endpoint of the trial was acute moist desquamation. Secondary endpoints included other parameters of acute skin toxicity (dermatitis, pruritus, oedema), chronic skin toxicity and cosmesis, quality of life, dose-volume parameters, cost and genetic predictors for toxicity. This manuscript only reports on acute skin toxicity and dose-volume parameters.

Sample size calculation for this phase II randomized trial was done assuming a $10 \%$ rate of acute moist desquamation with prone IMRT. A $15 \%$ rate of moist desquamation was considered to be unacceptable. Using the Wilson score confidence interval test, a minimum of 165 patients needed to be randomized. The aim was to include 170 patients ( 85 in each treatment arm).

Toxicity was measured by a radiation oncologist or a dedicated nurse, not blinded to the treatment arm, at baseline (on the day of radiotherapy start), once a week during radiotherapy and at 1014 days after treatment start. Desquamation was scored as: none, dry or moist. Dermatitis was scored using the Common Toxicity Criteria for Adverse Events (CTCAE) (http://ctep.cancer.gov/protocolDevelopment/electronic_applications/ctc.htm); pruritus and oedema as absent or present.

\section{Skin care}

Before the start of radiation treatment, patients were informed about preventive skin care measures: showering and not bathing, no rubbing on the skin, use of neutral soap. They were also asked not to use deodorant or any topical applications other than the ones prescribed by the research team. No preventive topical applications were used. If skin toxicity presented, a standard treatment protocol was used in both arms. Ureum 5\% in cold cream was prescribed for symptomatic erythema or dry skin. If cold cream was insufficient, a topical cream with methylprednisolone aceponate was applied (Advantan ${ }^{\mathrm{m}}$ ). Whenever moist desquamation presented, Mepilex ${ }^{\mathrm{m}}$ bandages were used.

\section{Statistics}

The SPSS version 23 statistical package was used for analysis. For comparison between both arms, the Fisher exact test was used for categorical variables, while the Mann-Whitney U test was used for continuous variables.

\section{Results}

Patient and treatment characteristics of the 167 analysed patients are shown in Table 1. Patients in the control arm were slightly older (mean age 59.5 years versus 55.7 years in the experimental arm, $p=0.028$ ). The other variables were not significantly different between treatment arms.

The analysis of dose parameters was done on 163 patients ( 80 patients in the control arm and 83 patients in the SIB arm). Reasons for excluding patients were electron boost (2), 3 different plans on 3 different CTs due to irreproducible anatomy changes (1) and changed treatment arm due to a machine breakdown (1). This latter patient was excluded from the toxicity analysis as well. She was randomized in the SeB arm, but after a treatment break, the responsible radiation oncologist decided to give the remaining 
Table 1

patient, tumour and treatment characteristics

\begin{tabular}{|c|c|c|c|c|}
\hline & & $\mathrm{SeB}($ mean $\pm \mathrm{SD})$ & $\mathrm{SIB}($ mean $\pm \mathrm{SD})$ & $p$-Value \\
\hline Body mass index & & $26.4 \pm 4.26$ & $25.2 \pm 4.01$ & .062 \\
\hline Age (years) & & $59.5 \pm 10.92$ & $55.7 \pm 10.54$ & .028 \\
\hline Breast volume (cc) & & $833.2 \pm 397.06$ & $741.67 \pm 374.99$ & .108 \\
\hline \multirow[t]{2}{*}{ Boost volume (cc) } & & $42.3 \pm 27.51$ & $39.8 \pm 31.09$ & .407 \\
\hline & & $\operatorname{SeB}(N)$ & $\operatorname{SIB}(N)$ & $p$-Value \\
\hline \multirow[t]{2}{*}{ Localization } & Left & 37 & 44 & \\
\hline & Right & 47 & 39 & .280 \\
\hline \multirow[t]{2}{*}{ Lymph node dissection } & No & 80 & 78 & \\
\hline & Yes & 4 & 5 & .746 \\
\hline \multirow[t]{2}{*}{ Positive resection margin } & No & 80 & 75 & \\
\hline & Yes & 4 & 8 & .248 \\
\hline \multirow[t]{2}{*}{ Hormone therapy } & No & 14 & 11 & \\
\hline & Yes & 70 & 72 & .665 \\
\hline \multirow[t]{2}{*}{ Chemotherapy } & No & 52 & 50 & \\
\hline & Yes & 32 & 33 & .875 \\
\hline \multirow[t]{2}{*}{ Trastuzumab } & No & 75 & 71 & \\
\hline & Yes & 9 & 12 & .493 \\
\hline
\end{tabular}

Abbreviations: $\mathrm{SeB}=$ sequential boost, $\mathrm{SIB}=$ simultaneous integrated boost, $\mathrm{SD}=$ standard deviation, $N=$ number of patients.

fractions with a SIB-technique to preserve the overall treatment time.

Dosimetry results can be found on the journal's website in a Supplementary table. The preset D95 and D05 objectives were met for the $\mathrm{CTV}_{\text {boost }}$ for both treatment arms. When comparing EQD2 for D02 and D05 of the optim structure, significantly higher doses were found in the SeB group, both for an $\alpha / \beta$ value of $10 \mathrm{~Gy}$ and $3 \mathrm{~Gy}(p<.001)$. Dose distributions for a typical SeB and SIB plan are shown in Fig. 3. The isodoses of the SeB plan represent the summation of the plan for WBI and the plan for boost irradiation.

In both arms, 6/83 patients developed moist desquamation. Grade $2 / 3$ dermatitis was significantly more frequent in the SeB arm (38/83 vs 24/83 patients, $p=0.037)$. In the SIB and SeB arm, respectively, 36 patients (43\%) and 51 patients (61\%) developed pruritus $(p=0.015)$. The incidence of oedema was lower in the SIB arm (59 vs 68 patients), but not statistically significant $(p=0.071)$.

Fig. 4 shows the evolution of dermatitis, desquamation, pruritus and oedema over time. In both groups, the incidence of toxicity was highest in the last week of treatment. At the first follow-up visit 10 to 14 days after radiation treatment, the number of patients presenting with no toxicity was increased in both arms. Grade 2 dermatitis didn't appear until the 3rd week of treatment, reaching the highest incidence in week 4 in the sequential boost arm and in week 3 in the SIB arm. There was one patient in the SIB arm that had developed a grade 3 dermatitis at the first follow-up visit after radiotherapy. During the last week of treatment, patchy moist desquamation was already present and this aggravated to confluent moist desquamation in the first week after treatment.

\section{Discussion}

Strong evidence now exists that breast cancer cells are as sensitive to fraction size as healthy tissues [25], justifying the use of hypofractionation schemes lowering the total dose but increasing the dose per fraction. WBI in 15-16 fractions is standard practice in the United Kingdom for several years now. After publication of the 10 year results of the UK and Canadian randomized trials, showing excellent efficacy and less long-term side effects of these schemes compared to more protracted schemes [1,2], the use of moderate hypofractionation is increasing worldwide. The reduction in late toxicity might be explained by the reduction of the total dose. Another radiobiological advantage is the shortened overall treatment time, possibly improving disease free survival [1]. Of course, shorter treatment courses with less transportation to the radiotherapy department are less stressful to the patient and increase the number of available treatment slots for other patients.

Boost irradiation is again prolonging radiotherapy treatment and using a SIB is the next logical step to restrict the overall treatment time. From a technical point of view, SIB irradiation is not that challenging, but a higher dose per fraction is administered to a part of the breast, which might increase the incidence of acute and late toxicity. On the other hand, better dose conformity can be reached if doses to the whole breast and to the boost volume are optimized simultaneously. Less dose spilling and improved dose conformity with a SIB have been reported $[5,6]$. Another drawback of planning performed sequentially is that hot spots in the first plan can coincide with hot spots in the second (boost) plan. Modern treatment planning systems allow taking the dose delivered by the WBI plan into account when planning the boost, but setup differences or anatomy changes are not taken into account.

Two large non-comparative trials report acceptable toxicity of supine WBI combined with a SIB in 25 or 28 fractions [26,27]. In the Dutch trial, toxicity and cosmetic outcome are not impaired after 30 months in 436 patients treated with 3D conformal radiotherapy with SIB [27]. In the UK trial, retrospective review of 354 patients treated with SIB-IMRT showed favourable acute toxicity and 3-year cosmesis [26]. These data are confirmed by several smaller non-comparative studies using different fractionation schemes $[10,11,28-31]$. One of the pioneers of prone breast radiation therapy is the New York University School of Medicine. They have published the 5-year results of 404 patients treated in prone position with a 15 fractions schedule of $40.5 \mathrm{~Gy}$ to the whole breast and a concomitant boost of $0.5 \mathrm{~Gy}$ per fraction to a total dose of 48 Gy [15]. Very limited numbers of more than grade 1 toxicity were observed with about $80 \%$ of patients judging the final cosmetic result as good or excellent.

Comparative data are scarce. Only 2 randomized controlled trials were found. Very recently, the New York University School of Medicine reported on 400 prone-treated patients randomized between a daily concomitant boost and a weekly boost combined with a hypofractionation scheme [13]. Although, the weekly boost is not really a sequential boost, the trial has some similarities to our study, the most important being the fractionation schedule ( $40.05 \mathrm{~Gy}$ to the breast with a SIB of $47.55 \mathrm{~Gy}$ ). No difference was seen in acute or late toxicity [13] with similar quality of life outcomes [32]. The second randomized trial does not allow to draw 


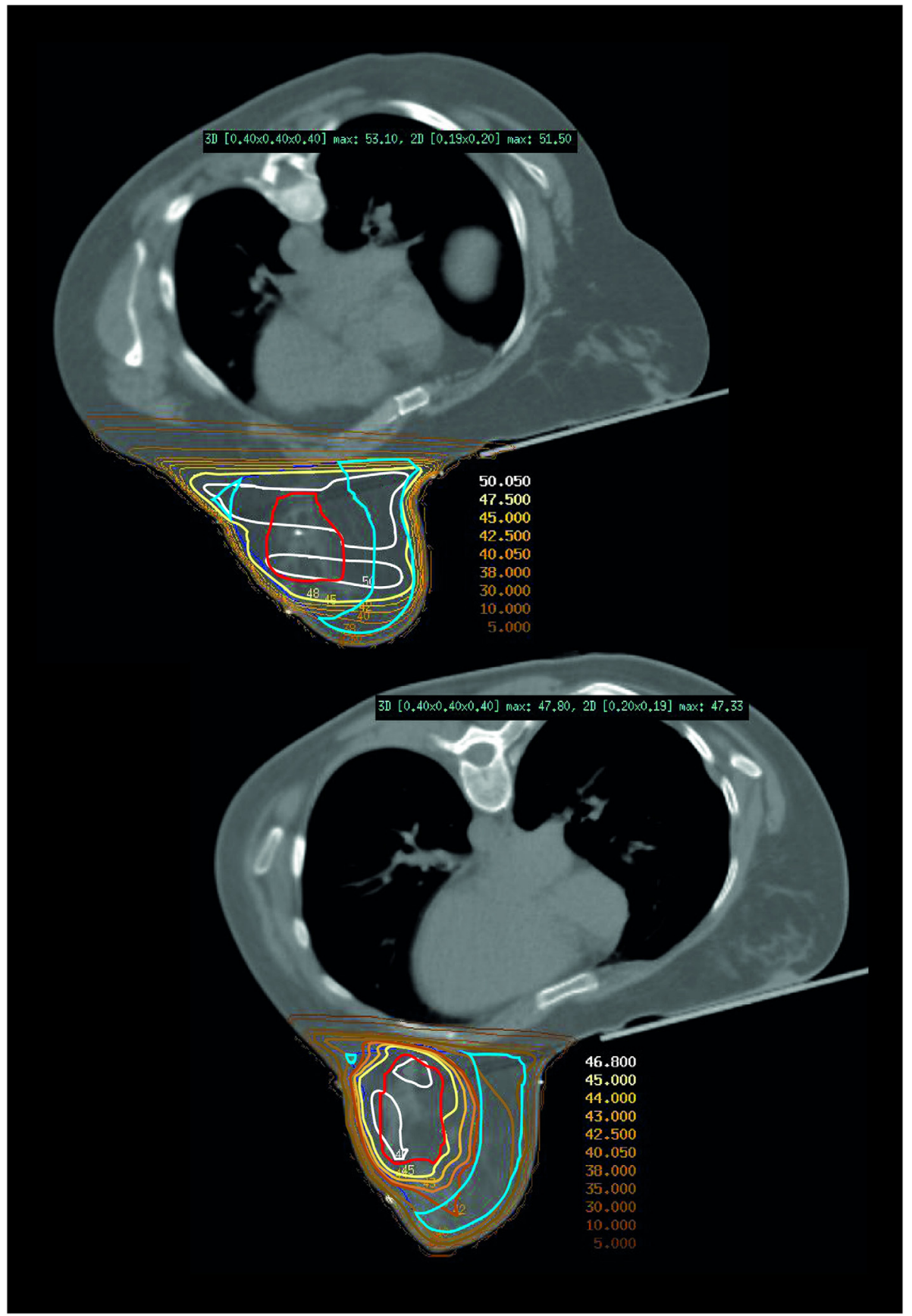

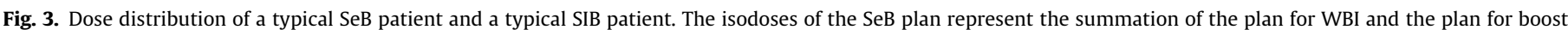

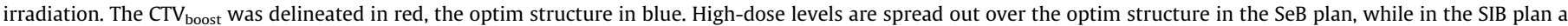
dose gradient is seen between the $\mathrm{CTV}_{\text {boost }}$ and the optim structure.

conclusions on the effect of a SIB since other factors differed between the SIB and SeB arm [12]. In the control arm, patients were treated with a normofractionation scheme of $50 \mathrm{~Gy}$ in 25 fractions with a SeB of $16 \mathrm{~Gy}$ in 8 fractions using conventional radiotherapy. In the SIB arm, patients were treated with a hypofractionation scheme of 15 times $2.8-3.4 \mathrm{~Gy}$ using helical tomotherapy. After 2 years, the incidence of toxicity was reduced from $60 \%$ to $30 \%$ in the SIB arm; heart and lung function were comparable between both arms. Lansu et al. retrospectively investigated the influence of SIB, hypofractionation and oncoplastic surgery on cosmetic outcome in 125 patients [8]. SIB did not have an influence on cosmetic outcome, but had a favourable influence on quality of life. Another non-randomized prospective trial in 60 patients showed comparable toxicity of SIB and SeB after 7 months [9].

The aim of this phase II randomized controlled trial was to evaluate whether the combination of a SIB with prone hypofractionated WBI would increase acute toxicity compared to the routinely used hypofractionation scheme of $40.05 \mathrm{~Gy} / 15$ fractions plus a SeB of $10 \mathrm{~Gy} / 4$ fractions or $14.88 \mathrm{~Gy} / 6$ fractions. Using an $\alpha / \beta$ value of $3.6 \mathrm{~Gy}$ as proposed by Yarnold [25] resulted in a 15 fractions schedule with a SIB of $3.12 \mathrm{~Gy}$ or $3.33 \mathrm{~Gy}$ per fraction. The rate of moist desquamation, the primary endpoint, was not different between both treatment arms. In both arms, 7\% of patients developed moist desquamation, which is lower than the anticipated $10 \%$ used for the sample size calculation. If the actual rate 
Sequential boost
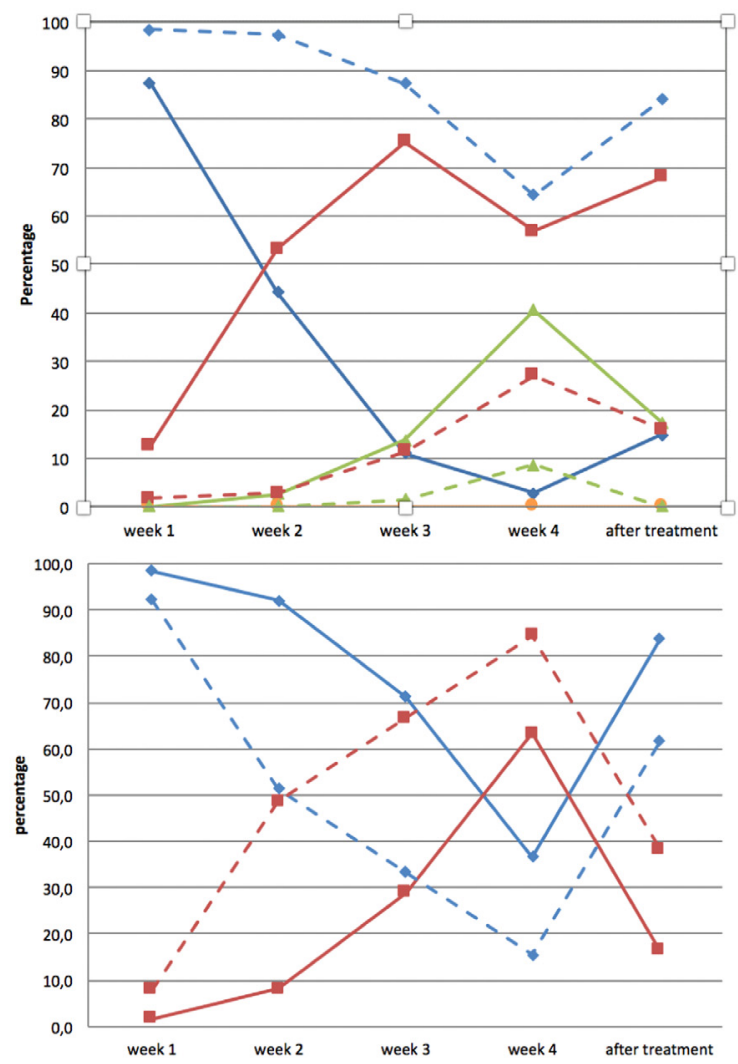

SIB
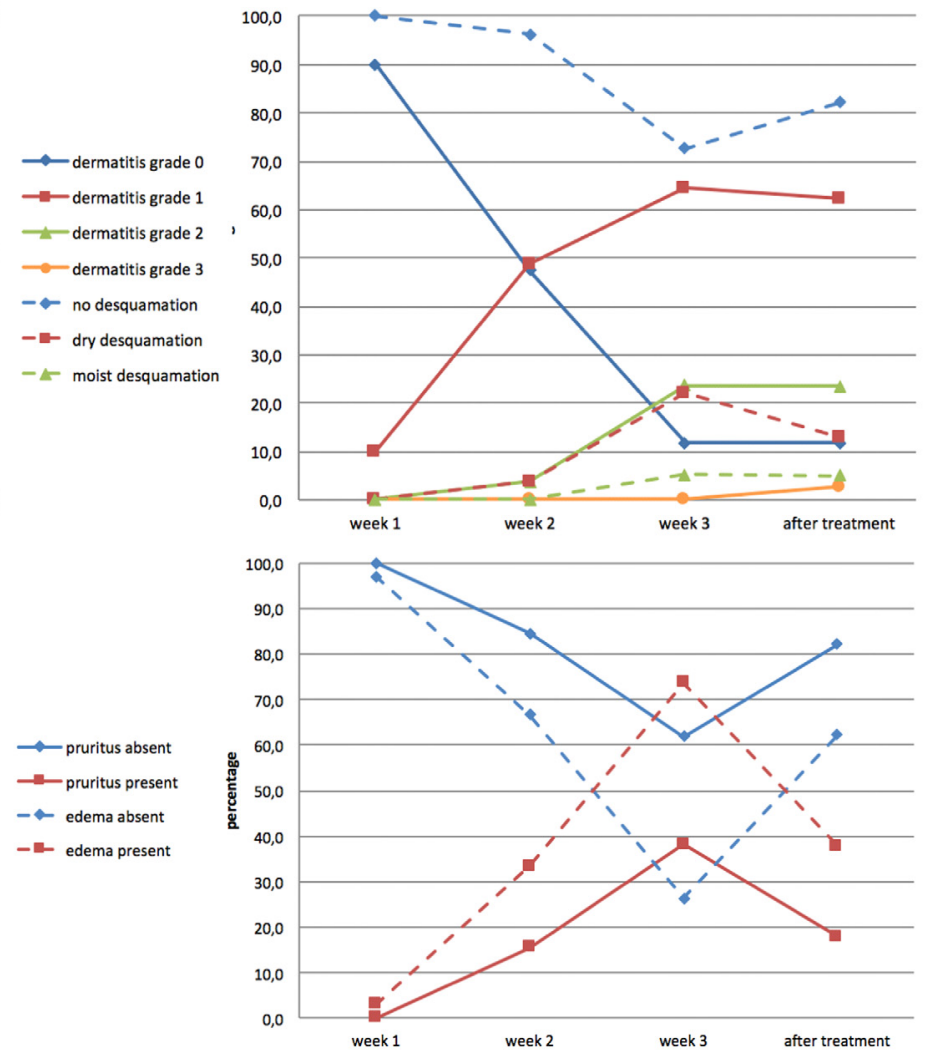

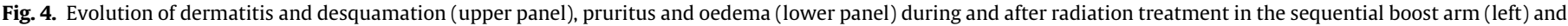
the simultaneous integrated boost (SIB) arm (right).

of moist desquamation was used in the sample size calculation, only 135 patients needed to be randomized to obtain the same power. The incidence of grade $2 / 3$ dermatitis and pruritus were reduced by one third in the SIB arm $(p=0.037$ and $p=0.015$, respectively). The 2 patients in the SeB arm that received an electron boost developed no more than grade 1 toxicity.

The favourable acute toxicity profile in the SIB group is consistent with the dosimetry results, showing a significantly lower D02 and D05 in this patient group $(p<.001)$. This is not solely explained by the difference in fractionation schedule. When an $\alpha / \beta$ of $10 \mathrm{~Gy}$ is used for acute side effects, a dose of $46.8 \mathrm{~Gy}$ in 15 fractions corresponds with an EQD2 of $51.2 \mathrm{~Gy}$ which is about $1.5 \mathrm{~Gy}$ lower than the $52.7 \mathrm{~Gy}$ calculated for a schedule of $40.05 \mathrm{~Gy}$ in 15 fractions followed by a boost of $10 \mathrm{~Gy}$ in 4 fractions. The differences in D02 and D05 observed in this study are much larger; the doses are about $4 \mathrm{~Gy}$ lower in the SIB arm. Using an $\alpha / \beta$ of $3 \mathrm{~Gy}$ for late side effects, this corresponds with an EQD2 of $57.3 \mathrm{~Gy}$ in the SIB arm, which is about $1 \mathrm{~Gy}$ higher than the EQD2 of $56.4 \mathrm{~Gy}$ in the SeB arm. Yet, the calculated D02 and D05 are lower in the SIB group even if an $\alpha / \beta$ of $3 \mathrm{~Gy}$ is used (Supplementary table). Fig. 3 nicely illustrates the difference in dose distribution between a $\mathrm{SeB}$ and a SIB plan. The high-isodose lines in the SIB patient are confined to the boost volume with dose decrease in the first $2 \mathrm{~cm}$ around the $\mathrm{CTV}_{\text {boost, }}$ whereas in the SeB patient, high dose regions are spread out over a larger part of the breast on the transverse slice. There were no significant differences observed in EQD2 doses for heart and ipsilateral_lung.

The first follow-up visit after treatment was set at 10-14 days after the last radiation session based on our experience with the 19-fractions schedule. The next follow-up visit was scheduled at 6 months to limit the burden for the patients. However, patients were advised to contact the investigators if toxicity got worse. In that case they were invited for a follow-up visit. There is a chance that toxicity presenting later than 10-14 days has been missed, but Fig. 4 is reassuring. At last follow-up the frequency of pruritus and oedema was declining and more case of grade 0 dermatitis were seen. The evolution of grade 2 dermatitis and moist desquamation was, however, different in both arms: while it was declining in the $\mathrm{SeB}$ arm after treatment, the incidence was comparable in week 3 and after treatment in the SIB arm. The fact that during boost delivery in the SeB group (week 4 of treatment), the whole-breast is no longer irradiated might be a possible explanation.

We acknowledge that the scoring of toxicity in this study has some limitations. First, the treatment arm was not blinded to the observer scoring toxicity. Second, grading of toxicity is often subjective. We tried to counter this by choosing a more objective endpoint, i.e. moist desquamation, as the primary endpoint. The difference between grade 1 and grade 2 dermatitis, i.e. faint erythema versus moderate to brisk erythema, is far more subjective. For the same reason, other endpoints like fatigue, pain, pruritus and oedema were dichotomized to present or absent. Evaluation of toxicity was done either by a dedicated study nurse (AVG) or a limited number of radiation oncologists (FL, CM, TM, PB or LV), all receiving training on toxicity scoring prior to the study.

Long term outcome results of SIB techniques for breast radiotherapy have to be awaited. Five-year local control seems promising $[14,15]$, but randomized data are not available. Since the same EQD2 is delivered in a shorter time span, there is, however, no 
reason to assume that local control or overall survival will be worse after a SIB than after a SeB. Late skin toxicity and cosmesis will further be observed in this patient cohort.

\section{Conclusion}

No difference was observed between treatment arms in the rate of moist desquamation, the primary endpoint of the trial. A SIB in combination with prone hypofractionated WBI appears to be a safe treatment regarding acute toxicity, but long-term data have to be awaited.

\section{Conflict of interest statement}

The authors report no conflict of interest.

\section{Acknowledgements}

This work was financed through Cancer Plan Action 29 (project 015 and 008) by the Federal Public Service of Health, Food Chain Safety and Environment, Belgium. Liv Veldeman is funded by a grant of Kom op tegen Kanker (Clinical Mandate). Christel Monten and Tom Vercauteren are recipients of a grant from the Clinical Research Fund of Ghent University Hospital. Special thanks to Karolien Roelants who was responsible for the artwork.

\section{Appendix A. Supplementary data}

Supplementary data associated with this article can be found, in the online version, at http://dx.doi.org/10.1016/j.radonc.2016.12. 023.

\section{References}

[1] Haviland JS, Owen JR, Dewar JA, Agrawal RK, Barrett J, Barrett-Lee PJ, et al. The UK Standardisation of Breast Radiotherapy (START) trials of radiotherapy hypofractionation for treatment of early breast cancer: 10-year follow-up results of two randomised controlled trials. Lancet Oncol 2013;14:1086-94.

[2] Whelan TJ, Pignol JP, Levine MN, Julian JA, MacKenzie R, Parpia S, et al. Longterm results of hypofractionated radiation therapy for breast cancer. N Engl J Med 2010;362:513-20.

[3] Bartelink H, Maingon P, Poortmans P, Weltens C, Fourquet A, Jager J, et al, Whole-breast irradiation with or without a boost for patients treated with breast-conserving surgery for early breast cancer: 20-year follow-up of a randomised phase 3 trial. Lancet Oncol 2015;16:47-56.

[4] Aly MM, Abo-Madyan Y, Jahnke L, Wenz F, Glatting G. Comparison of breast sequential and simultaneous integrated boost using the biologically effective dose volume histogram (BEDVH). Radiat Oncol 2016;11:16.

[5] Van Parijs H, Reynders T, Heuninckx K, Verellen D, Storme G, De Ridder M. Breast conserving treatment for breast cancer: dosimetric comparison of sequential versus simultaneous integrated photon boost. Biomed Res Int 2014;2014:827475.

[6] Singla R, King S, Albuquerque K, Creech S, Dogan N. Simultaneous-integrated boost intensity-modulated radiation therapy (SIB-IMRT) in the treatment of early-stage left-sided breast carcinoma. Med Dosim 2006;31:190-6.

[7] Guerrero M, Li XA, Earl MA, Sarfaraz M, Kiggundu E. Simultaneous integrated boost for breast cancer using IMRT: a radiobiological and treatment planning study. Int J Radiat Oncol Biol Phys 2004;59:1513-22.

[8] Lansu JT, Essers M, Voogd AC, Luiten EJ, Buijs C, Groenendaal N, et al. The influence of simultaneous integrated boost, hypofractionation and oncoplastic surgery on cosmetic outcome and PROMs after breast conserving therapy. Eur J Surg Oncol 2015;41:1411-6.

[9] Raiyawa T, Lertbutsayanukul C, Rojpornpradit P. Late effects and cosmetic results of simultaneous integrated boost versus sequential boost after conventional irradiation in breast-conserving therapy; out come of 7 months follow-up. J Med Assoc Thai 2009;92:390-7.

[10] Scorsetti M, Alongi F, Fogliata A, Pentimalli S, Navarria P, Lobefalo F, et al. Phase I-II study of hypofractionated simultaneous integrated boost using volumetric modulated arc therapy for adjuvant radiation therapy in breast cancer patients: a report of feasibility and early toxicity results in the first 50 treatments. Radiat Oncol 2012;7:145.

[11] Dellas K, Vonthein R, Zimmer J, Dinges S, Boicev AD, Andreas P, et al. Hypofractionation with simultaneous integrated boost for early breast cancer: results of the German multicenter phase II trial (ARO-2010-01). Strahlenther Onkol 2014;190:646-53.

[12] Van Parijs H, Miedema G, Vinh-Hung V, Verbanck S, Adriaenssens N, Kerkhove $D$, et al. Short course radiotherapy with simultaneous integrated boost for stage I-II breast cancer, early toxicities of a randomized clinical trial. Radiat Oncol 2012;7:80.

[13] Cooper BT, Formenti-Ujlaki GF, Li X, Shin SM, Fenton-Kerimian M, Guth A, et al. Prospective randomized trial of prone accelerated intensity modulated breast radiation therapy with a daily versus weekly boost to the tumor bed. Int J Radiat Oncol Biol Phys 2016;95:571-8.

[14] Bantema-Joppe EJ, Vredeveld EJ, de Bock GH, Busz DM, Woltman-van Iersel M, Dolsma WV, et al. Five year outcomes of hypofractionated simultaneous integrated boost irradiation in breast conserving therapy; patterns of recurrence. Radiother Oncol 2013;108:269-72.

[15] Osa EO, DeWyngaert K, Roses D, Speyer J, Guth A, Axelrod D, et al. Prone breast intensity modulated radiation therapy: 5-year results. Int J Radiat Oncol Biol Phys 2014;89:899-906.

[16] Kirby AM, Evans PM, Donovan EM, Convery HM, Haviland JS, Yarnold JR. Prone versus supine positioning for whole and partial-breast radiotherapy: a comparison of non-target tissue dosimetry. Radiother Oncol 2010;96:178-84.

[17] Lymberis SC, Dewyngaert JK, Parhar P, Chhabra AM, Fenton-Kerimian M, Chang J, et al. Prospective assessment of optimal individual position (prone versus supine) for breast radiotherapy: volumetric and dosimetric correlations in 100 patients. Int J Radiat Oncol Biol Phys 2012;84:902-9.

[18] Formenti SC, DeWyngaert JK, Jozsef G, Goldberg JD. Prone vs supine positioning for breast cancer radiotherapy. JAMA 2012;308:861-3.

[19] Mulliez T, Veldeman L, van Greveling A, Speleers B, Sadeghi S, Berwouts D, et al. Hypofractionated whole breast irradiation for patients with large breasts: a randomized trial comparing prone and supine positions. Radiother Oncol 2013;108:203-8.

[20] Mulliez T, Veldeman L, Speleers B, Mahjoubi K, Remouchamps V, Van Greveling A, et al. Heart dose reduction by prone deep inspiration breath hold in left-sided breast irradiation. Radiother Oncol 2015;114:79-84.

[21] Veldeman L, Speleers B, Bakker M, Jacobs F, Coghe M, De Gersem W, et al Preliminary results on setup precision of prone-lateral patient positioning for whole breast irradiation. Int J Radiat Oncol Biol Phys 2010;78:111-8.

[22] Mulliez T, Speleers B, Madani I, De Gersem W, Veldeman L, De Neve W. Whole breast radiotherapy in prone and supine position: is there a place for multibeam IMRT? Radiat Oncol 2013;8:151.

[23] Vercauteren T, De Gersem W, Olteanu LA, Madani I, Duprez F, Berwouts D, et al. Deformation field validation and inversion applied to adaptive radiation therapy. Phys Med Biol 2013;58:5269-86.

[24] Holland R, Veling SH, Mravunac M, Hendriks JH. Histologic multifocality of Tis, T1-2 breast carcinomas. Implications for clinical trials of breast-conserving surgery. Cancer 1985;56:979-90.

[25] Yarnold J, Ashton A, Bliss J, Homewood J, Harper C, Hanson J, et al. Fractionation sensitivity and dose response of late adverse effects in the breast after radiotherapy for early breast cancer: long-term results of a randomised trial. Radiother Oncol 2005;75:9-17.

[26] McDonald MW, Godette KD, Whitaker DJ, Davis LW, Johnstone PA. Three-year outcomes of breast intensity-modulated radiation therapy with simultaneous integrated boost. Int J Radiat Oncol Biol Phys 2009;77:523-30.

[27] Bantema-Joppe EJ, Schilstra C, de Bock GH, Dolsma WV, Busz DM, Langendijk JA, et al. Simultaneous integrated boost irradiation after breast-conserving surgery: physician-rated toxicity and cosmetic outcome at 30 months' followup. Int J Radiat Oncol Biol Phys 2012;83:e471-7.

[28] Fiorentino A, Mazzola R, Ricchetti F, Giaj Levra N, Fersino S, Naccarato S, et al. Intensity modulated radiation therapy with simultaneous integrated boost in early breast cancer irradiation. Report of feasibility and preliminary toxicity. Cancer Radiother 2015;19:289-94.

[29] Lee HC, Kim SH, Suh YJ, Chung MJ, Kang DG, Choi HJ, et al. A prospective cohort study on postoperative radiotherapy with TomoDirect using simultaneous integrated boost technique in early breast cancer. Radiat Oncol 2014;9:244.

[30] Alford SL, Prassas GN, Vogelesang CR, Leggett HJ, Hamilton CS. Adjuvant breast radiotherapy using a simultaneous integrated boost: clinical and dosimetric perspectives. J Med Imaging Radiat Oncol 2013;57:222-9.

[31] Ghannam AA, Khedr RA. An accelerated hypofractionated schedule with a daily concomitant boost after breast conservation surgery: the feasibility and toxicity. J Egypt Natl Cancer Inst 2016;28:39-44.

[32] Finkel MA, Cooper BT, Li X, Fenton-Kerimian M, Goldberg JD, Formenti SC. Quality of life in women undergoing breast irradiation in a randomized, controlled clinical trial evaluating different tumor bed boost fractionations. Int J Radiat Oncol Biol Phys 2016;95:579-89. 